INPLASY

PROTOCOL

To cite: Garbarino et al.

Laparoscopic versus open surgery for gastric cancer in

Western countries: a

systematic review and metaanalysis of short- and long-

term outcomes. Inplasy

protocol 202150008. doi:

10.37766/inplasy2021.5.0008

Received: 01 May 2021

Published: 01 May 2021

Corresponding author:

Gianluca Costa

g.costa@unicampus.it

Author Affiliation:

University Campus Bio-

Medico, Rome, Italy.

Support: None.

Review Stage at time of this submission: The review has not yet started.

Conflicts of interest:

None declared.

\section{Laparoscopic versus open surgery for gastric cancer in Western countries: a systematic review and meta-analysis of short- and long-term outcomes}

Garbarino, GM1; Lucarini, A2; Laracca, GGM33; Piccolino, M4; Mercantini, P5; Costa, A6; La Vaccara, V7; Costa, G8.

Review question / Objective: The objective of this systematic review and meta-analysis is to merge all western studies comparing $L G$ and $O G$ available in literature in the attempt to increase the statistical power and level of evidence supporting the use of laparoscopic gastrectomy for the treatment of gastric cancer even in low- middle incidence countries.

Condition being studied: Studies reporting a comparison between laparoscopic and open approaches on adult patients undergoing gastrectomy for cancer will be the focus of this review. Studies including hybrid laparoscopic-robotic procedures or comparing robotic to laparoscopic gastrectomy will be excluded.

INPLASY registration number: This protocol was registered with the International Platform of Registered Systematic Review and Meta-Analysis Protocols (INPLASY) on 01 May 2021 and was last updated on 01 May 2021 (registration number INPLASY202150007).

\section{INTRODUCTION}

Review question / Objective: The objective of this systematic review and meta-analysis is to merge all western studies comparing LG and OG available in literature in the attempt to increase the statistical power and level of evidence supporting the use of laparoscopic gastrectomy for the treatment of gastric cancer even in low- middle incidence countries.

Condition being studied: Studies reporting a comparison between laparoscopic and open approaches on adult patients undergoing gastrectomy for cancer will be 
the focus of this review. Studies including hybrid laparoscopic-robotic procedures or comparing robotic to laparoscopic gastrectomy will be excluded.

\section{METHODS}

Search strategy: A systematic review will be accomplished according to the PRISMA statement in order to identify articles comparing laparoscopic and open surgery in the treatment of gastric cancer. The following keywords and/or medical subject heading (MeSH) terms will be used in various combinations: "gastric cancer", "laparoscopy", "western", "west", "Europe", "US", and "USA". A manual search will also be performed in Google Scholar and in the reference lists of relevant articles to find potential additional studies. The search will be carried out by using English language terms but no restriction will be adopted to exclude any paper neither by language nor by study type.

Participant or population: Adult patients undergoing surgery for gastric cancer.

Intervention: Laparoscopic gastrectomy for gastric cancer.

Comparator: Open gastrectomy for gastric cancer.

Study designs to be included: All type.

Eligibility criteria: Only studies reporting a comparison between laparoscopic and open approach on adult patients undergoing gastrectomy for cancer will be considered. At least one per-operative outcome of interest should be reported with or without any follow-up period including overall survival (OS) and/or disease-free survival (DFS).

Information sources: A literature search will be carried out through MEDLINE (PubMed), Embase, WebOfScience, and Scopus from January 1980 to 31 December 2020. A manual search will also be performed in Google Scholar and in the reference lists of relevant articles to find potential additional studies.

Main outcome(s): Operative time, LN harvested, Blood loss, Analgesic requirement, Time to first flatus, Time to oral intake, Overall morbidity, Major complications (Clavien-Dindo III-IV), Length of stay (LOS), Mortality, 3-year overall survival, 5-year overall survival. Categorical variables will be evaluated using the odds ratio (OR) and $95 \% \mathrm{Cl}$. Continuous variables will be analyzed by the weighted mean difference (WMD) and $95 \%$ confidence interval $(\mathrm{Cl})$. When variables are reported in the papers as median and range or interquartile range, they will be converted to mean and standard deviation (SD) according to Hozo. Hazard ratios (HRs) will be used to analyze time to event outcomes (OS and DFS). When the HRs and $95 \% \mathrm{Cl}$ are not provided in the studies, two authors (AC and VLV), following wellestablished methodologies, will extract data from Kaplan-Meier (KM) curves with GraphClick software $\mathbf{3 . 0}$ for Mac (ArizonaSoftware) and will estimate the HRs using on-line calculator (https: / / www.gigalculator.com/calculators/hazardratio-calculator.php).

Data management: Statistical analysis will be carried out using StataCorp2019 STATA Statistical Software: release 16 (College Station,TX: StataCorp LLC).

Quality assessment / Risk of bias analysis: The quality of the studies will be estimated by using the Newcastle-Ottawa Scale or Jadad's scale for RCTs when appropriate.

Strategy of data synthesis: Categorical variables will be evaluated using the odds ratio (OR) and $95 \% \mathrm{Cl}$. Continuous variables will be analyzed by the weighted mean difference (WMD) and $95 \%$ confidence interval (Cl). When variables are reported in the papers as median and range or interquartile range, they will be converted to mean and standard deviation (SD) according to Hozo. Hazard ratios (HRs) will be used to analyze time to event outcomes (OS and DFS). When the HRs and $95 \% \mathrm{Cl}$ are not provided in the studies, two 
authors (AC and VLV), following wellestablished methodologies, will extract data from Kaplan-Meier (KM) curves with GraphClick software $\mathbf{3 . 0}$ for Mac (ArizonaSoftware) and will estimate the HRs using on-Iine calculator (https: / / www.gigalculator.com/calculators/hazardratio-calculator.php).

Subgroup analysis: Subgroup analyses will be performed considering either the type of resection (Total and sub-total gastrectomy) and 5-year periods.

Sensitivity analysis: Heterogeneity between the studies will be assessed. When 12 value will be higher than $50 \%$, pooled estimates will be obtained using a random effects model. As regards to $p$ value of $Q$ index (chi-square test of heterogeneity) a $p<0.10$ was considered significant otherwise a conventional level of $p<0.05$ was accepted as statistically significant. Publication bias assessment will be performed by analyzing funnel plot asymmetry with Egger's test for continuous outcomes and with Harbord's and Peters's test for binary outcome.

Language: None.

Country(ies) involved: Italy.

Keywords: Gastric cancer, Laparoscopy, Western countries.

Dissemination plans: Papers.

Contributions of each author:

Author 1 - Giovanni Maria Garbarino Study conception, design, drafting manuscript.

Email: giovannimaria.garbarino@uniroma1.it

Author 2 - Alessio Lucarini - Literature search and data extraction.

Email: alessio.lucarini@uniroma1.it

Author 3 - Giovanni Guglielmo Laracca Literature search and data extraction.

Email: giovanni.lar@hotmail.it

Author 4 - Gianmarco Piccolino - Literature search and data extraction.

Email: gianmarco.piccolino@uniroma.it

Author 5 - Paolo Mercantini - Study conception and design, critical revision of manuscript.
Email: paolo.mercantini@uniroma.it

Author 6 - Alessandro Costa Management, analysis, and interpretation of data.

Email: alessandro.costa.16.10@gmail.com

Author 7 - Vincenzo La Vaccara Management, analysis, and interpretation of data.

Email: v.lavaccara@unicampus.it

Author 8 - Gianluca Costa - Study conception and design, statistical analysis, drafting of the manuscript.

Email: g.costa@unicampus.it 